\title{
Diagnóstico parcial da arborização viária sob rede elétrica na Regional Oeste de Minas Gerais
}

\author{
Altamir Fernandes de Oliveira ${ }^{1 *}$, José Aldo Alves Pereira² ${ }^{2}$ Silvério José Coelho², Gabriel de Assis Pereira² Lucélio Nativo da Assunção $^{3}$ \\ 1Universidade Federal dos Vales do Jequitinhonha e Mucuri, Câmpus Mucuri, Rua do Cruzeiro, n 01, Jardim São Paulo, CEP 39803-371, Teófilo Otoni, MG, Brasil \\ Universidade Federal de Lavras, Câmpus Universitário, C. P. 3037, CEP 37200-000, Lavras, MG, Brasil \\ ${ }^{3}$ Companhia Energética de Minas Gerais, Rua Padre Francisco Goulart, n 155, Centro, CEP 35582-000, Pains, MG, Brasil
}

\section{"Autor correspondente:}

altamirf83@gmail.com

Termos para indexação:

Árvore

Fiação elétrica

Gestão pública

Índices de diversidade

Poda

\author{
Index terms: \\ Tree \\ Electrical wiring \\ Public administration \\ Diversity indices \\ Pruning
}

Histórico do artigo:

Recebido em 16/07/2014

Aprovado em 30/12/2015

Publicado em 31/03/2016

doi: 10.4336/2016.pfb.36.85.749
Resumo - Este trabalho teve por objetivo realizar um diagnóstico parcial da arborização viária sob a rede de energia elétrica, em cinco cidades da Regional Oeste de Minas Gerais que mais apresentaram desligamentos de energia por causa de conflitos com árvores. Foram identificadas 186 árvores, distribuídas em 17 espécies de origem exótica e 30 espécies de origem nativa. As espécies mais frequentes foram Poincianella pluviosa (30,43\%), Ligustrum lucidum (10,86\%), Michelia champaca (6,52\%) e Schinus molle $(4,89 \%)$, que juntas perfizeram $52,7 \%$ das árvores avaliadas. A maior parte das árvores $(84,78 \%$ ) estava localizada em ruas com largura superior a $7 \mathrm{~m}$, sendo que $36,55 \%$ das árvores estavam plantadas em calçadas com largura mínima de $2 \mathrm{~m}$ e apresentaram altura da primeira bifurcação superior a $1,80 \mathrm{~m}$. As podas realizadas por causa de conflitos com a rede de energia equivaleram a 40,22\%. As árvores com altura maior ou igual a 6 m compuseram $72,83 \%$ da população amostral. Foi verificado que não há um plano adequado de manejo arbóreo-urbano sob a rede de energia, visto que a frequência de árvores se concentra em poucas espécies, que são em sua maioria de médio e grande porte.

\section{Partial diagnosis of street tree under power lines in West Regional of Minas Gerais}

\begin{abstract}
This work aimed to present a partial diagnosis of street tree under power grid in five cities in the West Region of Minas Gerais, which had more power outages because of conflicts with trees. We identified 186 trees, belonging to 17 species of exotic origin and 30 species of native origin. The most frequent species were Poincianella pluviosa (30.43\%), Ligustrum lucidum (10.86\%), Michelia champaca (6.52\%) and Schinus molle $(4.89 \%)$, which together amounted to $52.7 \%$ of the assessed trees. Most trees $(84.78 \%$ ) were located in streets wider than $7 \mathrm{~m}$, and $36.55 \%$ of the trees were planted in sidewalks with a minimum of $2 \mathrm{~m}$ wide. Those trees present ther first bifurcation at over $1.80 \mathrm{~m}$ above ground level. The pruning performed due to conflicts with the grid amounted $40.22 \%$. Trees with height equal or over $6 \mathrm{~m}$ represents $72.83 \%$ of the sampled population. It was observed that there is not an appropriate management plan of urban trees under the grid, as the trees frequency is concentrate on few species that were mostly medium and large size.
\end{abstract}




\section{Introdução}

A arborização urbana é uma das abordagens que, gradualmente, tem ganhado reconhecimento internacional. O termo arborização é utilizado para a ação ou para o resultado do plantio e da manutenção de árvores individuais ou em pequenos grupos (Magalhães, 2006), enquanto o termo floresta urbana se remete a um contexto de diversidade ambiental complexo, baseado na interconexão dos inúmeros componentes do sistema urbano (Driscoll, 2015). Os benefícios gerados pela presença de árvores no meio urbano podem ser inseridos em dois grupos, os ambientais e os de bem-estar social (Milano et al., 1992), dentre os quais destaca-se a amenização climática em virtude do sombreamento, a umidificação do ar em decorrência da constante transpiração (Paiva \& Gonçalves, 2002), a melhoria da qualidade do ar, redução da poluição atmosférica, proteção e direcionamento dos ventos (Companhia Energética de Minas Gerai, 2011) e redução dos níveis de ruídos provenientes de automóveis, equipamentos, indústrias e construções (Santos \& Teixeira, 2001; Dhzambov \& Dimitrova, 2015).

Planejar a implantação das árvores no meio urbano requer um processo cuidadoso, com atenção aos seus requisitos iniciais, bem como a devida manutenção (Companhia Energética de Minas Gerai, 2011). De acordo com Bobrowski (2011), o sucesso dos planos de arborização tem a ver com o seu planejamento, uma vez que evita a geração de danos, problemas e prejuízos futuros, caso seja feito adequadamente.

Logo, o plantio de árvores nas cidades deve ser feito levando-se em consideração o espaço físico disponível e as condições ambientais e antrópicas específicas. A prioridade pela seleção de espécies de baixo porte deve ser dada às vias públicas que contém redes de distribuição de energia elétrica, com a intenção de se evitarem conflitos futuros. Nesse caso, as espécies com possibilidade de condução de copa e crescimento lento também podem ser utilizadas, desde que com o devido respaldo técnico (Companhia Energética de Minas Gerai, 2011).

Segundo Milano (1987), são problemas recorrentes relativos à arborização urbana os danos à tubulação subterrânea, o levantamento de calçadas, a incompatibilidade das espécies com os devidos espaços disponíveis, bem como os conflitos com as redes elétricas. Sendo assim, faz-se necessário um olhar atento às relações entre árvores e redes elétricas, no intuito de se evitar acidentes com a população e bloqueios na distribuição de eletricidade. Por meio de informações coletadas na Companhia Energética de Minas Gerais (2013), apenas no ano de 2012, naquele Estado, foram relatadas 18.346 interrupções no fornecimento de energia, tendo como causa o conflito advindo pelas árvores no meio urbano.

Ressalta-se, ainda, a grande contribuição que os inventários da arborização urbana promovem às concessionárias e ao Poder Público, uma vez que permitem extrair informações cruciais como a identificação de espécies e de problemas e conflitos com os elementos urbanos, o que são essenciais para o direcionamento de ações de planejamento e manejo.

Diante disso, o objetivo deste trabalho foi realizar um diagnóstico parcial da arborização viária sob a rede de distribuição de energia elétrica, em cinco cidades da Regional Oeste de Minas Gerais, que de acordo com o ranking, obtiveram mais desligamento de energia elétrica relacionado a conflitos com espécies arbóreas nesta regional.

\section{Material e métodos}

\section{Seleção das cidades amostradas e coleta de dados}

De acordo com a divisão adotada pelo Instituto Brasileiro de Geografia e Estatística (IBGE), aquelas cidades compõem a Regional Oeste. O Estado de Minas é classificado em 12 mesorregiões, com o objetivo de elaborar políticas públicas e definir ações específicas para as regiões com determinadas atividades econômicas, sociais e tributárias, dentre outras. Foi selecionada a Regional Oeste, formada pelas cidades de Arcos, Divinópolis, Formiga, Itaúna e Pará de Minas (IBGE, 2010). O levantamento de cidades considerou os domínios fitogeográficos do Estado, na tentativa de estabelecer relação entre espécies nativas identificadas e sua ocorrência no respectivo domínio fitogeográfico.

Foram diagnosticados trechos de redes elétricas ou circuitos que continham árvores próximas. A seleção considerou os relatórios tabelados das cidades desta regional que mais apresentaram desligamentos de energia elétrica, relacionados ao conflito com árvores nos anos de 2011 e 2012, fornecidos em planilha eletrônica pela Companhia Energética de Minas Gerais (CEMIG).

Foram utilizadas as informações de duração de interrupção de energia, número e endereço dos dispositivos operados, número de consumidores e 
extensão em $\mathrm{km}$ dos circuitos elétricos. As cidades foram ordenadas, considerando suas respectivas durações de interrupção nos anos de 2011 e 2012.

\section{Diagnóstico quali-quantitativo parcial da arborização}

Os circuitos onde as espécies arbóreas foram avaliadas, apresentaram as seguintes características, segundo Companhia Energética de Minas Gerais (2011): média tensão, com chaves fusíveis e outros equipamentos operantes, também chamados de circuitos primários e que funcionam nas tensões de 13.800 a $34.500 \mathrm{v}$. Estes alimentam os transformadores, que convertem em tensões adequadas para uso residencial. A partir dessa informação, procurou-se identificar e localizar os circuitos para cada cidade. A melhor rota para os trabalhos de campo em cada cidade foi escolhida com o auxílio de técnicos da CEMIG, os quais ajudaram na análise e localização dos circuitos a serem amostrados. Inicialmente fez-se a análise visual de toda a extensão do circuito elétrico presente nas ruas e/ou avenidas, a fim de verificar a quantidade de árvores a serem avaliadas. Quando não foram encontrados indivíduos arbóreos no circuito, selecionou-se o mais próximo com a presença de pelo menos um espécime. Estes circuitos eram desprovidos de árvores, em decorrência da sua remoção por estarem próximas à rede elétrica e causarem desligamentos.

As informações foram obtidas, por meio de acesso autorizado pela CEMIG, em um sistema denominado Gemini, que permite a busca e análise de dados de circuitos elétricos, mapas e outros dados, como a quantidade de unidades consumidoras por circuito, informação bastante útil para ser relacionada às espécies ou indivíduos arbóreos identificados em cada cidade. Foi realizado o censo de todas as mudas e árvores por circuito. A quantidade total de árvores avaliadas por cidade resulta da soma dos registros em cada circuito percorrido.

A avaliação qualitativa baseou-se na identificação, localização, análise estrutural e caracterização do indivíduo arbóreo.

\section{Identificação e localização}

Foram determinados os seguintes parâmetros: cidade, data, número da árvore, nome comum, nome científico, família, endereço, identificação da rede de média tensão (trifásica, monofásica, isolada, protegida, nua ou ausente), rede de baixa tensão (trifásica, monofásica, isolada, nua ou ausente) e localização (praça, internalote, calçada-rede, calçada-oposta, canteiro central ou rua).

Para as espécies sem possibilidade de identificação imediata, arquivaram-se fotografias da árvore inteira, do tronco, das folhas, dos frutos e flores, quando possível, com o objetivo de auxiliar na identificação das espécies por especialistas da Universidade Federal de Lavras (UFLA), da Universidade Federal de Minas Gerais (UFMG) e da CEMIG.

Para certificar a identificação, foi promovida a conferência dos nomes científicos com as sinonímias substituídas pelos nomes aceitos, consultando banco de dados e bibliografias, conforme protocolo de Cupertino \& Eisenlohr (2013).

Posteriormente, identificou-se a origem das espécies, se nativas ou exóticas, consultando-se bibliografias específicas, bem como a altura potencial, segundo Aguirre Júnior \& Lima (2007), Araujo et al. (2012) e Melazo \& Nishiyama (2010), que estratificam as espécies arbóreo urbanas em pequeno porte $(\mathrm{P} \leq 6 \mathrm{~m})$, médio porte $(6 \mathrm{~m}<\mathrm{M} \leq 10 \mathrm{~m})$ e grande porte $(\mathrm{G}>10 \mathrm{~m})$, pois essas são classes que possuem melhor correlação com as alturas mínimas da fiação elétrica (Companhia Energética de Minas Gerais, 2002), para os diversos contextos na área urbana, nos municípios abrangidos pelo sistema elétrico da CEMIG.

Foi feita a atualização dos nomes científicos utilizando-se o banco de dados da Lista de Espécies da Flora do Brasil (Lista..., 2012) e o The Plant List (The Plant..., 2013).

\section{Caracterização do indivíduo arbóreo}

Para a análise estrutural, os elementos analisados foram: largura da rua, largura do passeio e distância da árvore ao poste, todos mensurados em m.

Para a caracterização do indivíduo arbóreo, considerouse a dimensão da copa $(\mathrm{m})$, fragmentada em raios (Figura 1) (em que R1 é o raio da projeção da copa em direção à casa/terreno/muro; R2 a projeção para o lado direito; R3 a projeção para a pista/sarjeta e R4 a projeção para o lado esquerdo); a altura da copa; a copa que aproxima ou não nas redes de baixa e média tensão; o tronco inclinado ou normal/ereto, com a presença de rachaduras/cavidades, cupins com sintomas de doenças ou sinais de fogo; a modalidade de poda (condução/formação, unilateral e destopa; "em V" ou inexistente); motivo da poda (sinalização/placas, semáforo, iluminação pública e edificação ou poste/rede) 
e sistema radicular (normal, exposto, com levantamento de calçada/pista, podado/seccionado, com sinais de apodrecimento, de fogo ou sem possibilidade de avaliar).

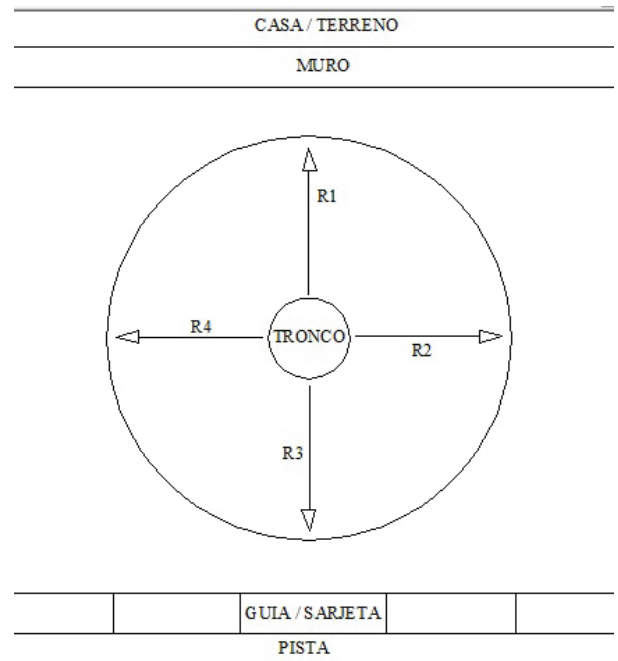

Figura 1. Esquema das projeções da copa (R1, R2, R3 e R4), para caracterização do indivíduo arbóreo.

\section{Análise dos dados}

Posteriormente aos trabalhos de campo, compôs-se uma planilha eletrônica com todas as fichas das árvores avaliadas, que resultaram em um banco de dados com os componentes coletados.

A relação completa das espécies encontradas por cidade foi elaborada, com o cálculo das respectivas frequências relativas (FR) e frequências acumuladas (FAc).

Estes parâmetros permitiram realizar comparações, segundo porcentagem de indivíduos encontrados em cada cidade, bem como realizar análises de ordenamento das espécies. As comparações foram realizadas para os dados de composição florística, extensão de redes elétricas, tipos de poda executados, classes de altura e conflitos com a rede elétrica.

Os parâmetros de fitossociologia: índice de riqueza de Odum, diversidade de Shannon-Wiener e equabilidade ou uniformidade de Pielou, foram calculados segundo Pinto-Coelho (2000).

A porcentagem apresentada para cada problema fitossanitário foi obtida dividindo-se a quantidade de indivíduos afetados pela quantidade total de indivíduos amostrados no diagnóstico.
Os gráficos foram construídos com o auxílio do programa Graphpad Prism 4.0 para Windows Graphpad Prism, 2015), que forneceu resultados estatísticos em comparações realizadas pelos testes $\mathrm{t}$ e $\mathrm{F}$. $\mathrm{O}$ teste $\mathrm{t}$ foi utilizado para análises de semelhança entre os índices de riqueza de Odum e de diversidade de espécies de Shannon-Wiener, calculados para os circuitos amostrados e entre $\mathrm{km}$ de redes elétricas das cidades amostradas e a população total, segundo IBGE (2010).

\section{Resultados e discussão}

As cidades avaliadas se localizam em zonas de Mata Atlântica e Cerrado (Minas Gerais, 2013), informações que são demasiadamente úteis ao planejamento e manejo da arborização urbana. A preferência por espécies nativas desses dois domínios fitogeográficos deve ser dada para a composição florística dos trabalhos em arborização urbana da região.

Dentre as cidades avaliadas, Divinópolis foi a maior, caracterizada como Polo Regional, com 213.016 hab. e densidade demográfica de 300,82 hab. $\mathrm{km}^{-2}$, seguida de Itaúna, contando $85.463 \mathrm{hab}$. (172,39 hab. $\mathrm{km}^{-2}$ ), Pará de Minas, contando 84.215 hab. (152,77 hab. $\left.\mathrm{km}^{-2}\right)$, Formiga, contando 65.128 hab. (43,36 hab. $\left.\mathrm{km}^{-2}\right)$ e Arcos, contando 36.597 hab. (71,78 hab. $\mathrm{km}^{-2}$ ) (IBGE, 2010).

A análise quanto ao porte das cidades amostradas, considerando o tamanho e potencial de crescimento da população, e o comprimento das redes de energia elétrica mostrou uma correlação significativa $\left(\mathrm{R}^{2}=0,9950\right.$, $\alpha=170,4 \pm 6,954$ e valor de $p=0,0001$, pelo teste $F$ ), o que é um sinal para que sejam elaborados planos de manejo da arborização urbana que considerem o plantio de espécies corretas nos devidos locais, adequadas ao convívio com a fiação. Logo, a consciência de que a pressão urbana sobre as árvores é crescente pode induzir ao incremento da biodiversidade local, proporcionando os vários benefícios que as árvores trazem ao seu entorno, bem como levam ao crescimento sustentável das cidades.

Nessa regional foram encontradas 186 árvores, divididas em 17 espécies de origem exótica e 30 de origem nativa (Tabela 1 ).

A família mais representativa foi Fabaceae, perfazendo $42,5 \%$ da população amostral, seguida de Oleaceae $(7,6 \%)$ e Bignoniaceae $(6,5 \%)$. As espécies mais 
frequentes foram Poincianella pluviosa (30,4\%), Ligustrum lucidum (10,9\%), Michelia champaca $(6,5 \%)$ e Schinus molle (4,9\%), com $52,7 \%$ do total. Dado o índice de $30 \%$ para a composição das famílias, e $10 \%$ para a composição das espécies na arborização urbana, segundo Santamour Junior (1990), o mesmo não está adequado nem para a família mais representativa (Fabaceae), nem para as duas espécies mais frequentes (Poincianella pluviosa e Ligustrum lucidum).

De acordo com Santamour Junior (1990), a composição de uma mesma família botânica em dada população arbórea urbana não deve ser maior do que $30 \%$, enquanto a porcentagem relativa ao gênero não deve ser maior que $20 \%$ e para composições relativas a espécies esse valor não deve ser maior que $10 \%$. A preocupação referente às porcentagens relatadas é a de que em caso de ataque de pragas, boa parte da população arbórea poderá ser afetada e, sem controles efetivos, muitos indivíduos deverão ser substituídos, o que aumenta os custos com a manutenção da arborização daquele município. Um plano de manejo satisfatório deve contemplar os referidos índices bem como atentar para o plantio conveniente de espécies que são compatíveis com os sistemas elétricos.

Nas cidades de Arcos e Pará de Minas a espécie Poincianella pluviosa foi a mais frequente, com $56,3 \%$ e $51,6 \%$ do total de indivíduos avaliados, respectivamente. Na cidade de Divinópolis Ligustrum lucidum correspondeu, sozinha, a 50,0\% da população amostral. Em Formiga, apesar de as dez espécies mais frequentes corresponderem a 53,5\% do total, Leucaena leucocephala e Schinus molle se destacaram. Por fim, em Itaúna Poincianella pluviosa e Schinus molle apresentaram o índice de $57,1 \%$, juntas.

Tabela 1. Composição florística obtida por meio do diagnóstico parcial da arborização viária sob rede elétrica da regional Oeste de Minas Gerais.

\begin{tabular}{|c|c|c|c|c|c|c|}
\hline ARCOS: S (7) & FAMÍLIA & Or. & Pr. & $\begin{array}{c}N \\
(48)\end{array}$ & $\begin{array}{l}\text { FR } \\
(\%)\end{array}$ & $\begin{array}{l}\text { FAc } \\
(\%)\end{array}$ \\
\hline Poincianella pluviosa (DC.) L. P. Queiroz - Sibipiruna & Fabaceae & $\mathrm{N}$ & G & 27 & 56,3 & 56,3 \\
\hline Michelia champaca L. - Magnólia & Magnoliaceae & $\mathrm{E}$ & M & 12 & 25,0 & 81,3 \\
\hline Licania tomentosa (Benth.) Fritsch - Oiti & Chrysobalanaceae & $\mathrm{N}$ & $\mathrm{G}$ & 3 & 6,3 & 87,5 \\
\hline Bauhinia forficata Link - Mororó-de-espinho & Fabaceae & $\mathrm{N}$ & $\mathrm{G}$ & 2 & 4,2 & 91,7 \\
\hline Murraya paniculata (L.) Jacq. - Murta & Rutaceae & $\mathrm{E}$ & $\mathrm{P}$ & 2 & 4,2 & 95,8 \\
\hline Ligustrum lucidum W. T. Aiton - Alfeneiro & Oleaceae & $\mathrm{E}$ & $\mathrm{P}$ & 1 & 2,1 & 97,9 \\
\hline Nerium oleander L. - Espirradeira & Apocynaceae & $\mathrm{E}$ & $\mathrm{P}$ & 1 & 2,1 & 100,0 \\
\hline DIVINÓPOLIS: S (12) & FAMÍLIA & Or. & Pr. & $\begin{array}{c}N \\
(36)\end{array}$ & $\begin{array}{l}\text { FR } \\
(\%)\end{array}$ & $\begin{array}{l}\text { FAc } \\
(\%)\end{array}$ \\
\hline Ligustrum lucidum W. T. Aiton - Alfeneiro & Oleaceae & $\mathrm{E}$ & $\mathrm{M}$ & 18 & 50,0 & 50,0 \\
\hline Tecoma stans (L.) Juss ex Kunth - Ipê-mirim & Bignoniaceae & $\mathrm{E}$ & M & 5 & 13,9 & 63,9 \\
\hline Lagerstroemia indica (L.) Pers. - Resedá & Lythraceae & $\mathrm{E}$ & $\mathrm{P}$ & 3 & 8,3 & 72,2 \\
\hline Tibouchina granulosa (Desr.) Cogn. - Quaresmeira & Melastomataceae & $\mathrm{N}$ & $\mathrm{G}$ & 2 & 5,6 & 77,8 \\
\hline Lagerstroemia speciosa Pers. - Escumilha-africana & Lythraceae & $\mathrm{E}$ & M & 1 & 2,8 & 80,6 \\
\hline Caesalpinia pulcherrima (L.) Sw. - Flamboyant-mirim & Fabaceae & $\mathrm{E}$ & $\mathrm{P}$ & 1 & 2,8 & 83,3 \\
\hline Jacaranda mimosifolia D. Don. - Jacarandá-mimoso & Bignoniaceae & $\mathrm{E}$ & $\mathrm{G}$ & 1 & 2,8 & 86,1 \\
\hline Murraya paniculata (L.) Jacq. - Murta & Rutaceae & $\mathrm{E}$ & $\mathrm{P}$ & 1 & 2,8 & 88,9 \\
\hline Licania tomentosa (Benth.) Fritsch - Oiti & Chrysobalanaceae & $\mathrm{N}$ & $\mathrm{G}$ & 1 & 2,8 & 91,7 \\
\hline Tibouchina candolleana (Mart. ex DC.) Cogn. - Quaresmeira & Melastomataceae & $\mathrm{N}$ & $\mathrm{M}$ & 1 & 2,8 & 94,4 \\
\hline Terminalia catappa L. - Sete-copas & Combretaceae & $\mathrm{E}$ & $\mathrm{G}$ & 1 & 2,8 & 97,2 \\
\hline Poincianella pluviosa (DC.) L. P. Queiroz - Sibipiruna & Fabaceae & $\mathrm{N}$ & G & 1 & 2,8 & 100,0 \\
\hline
\end{tabular}


Tabela 1. continuação.

\begin{tabular}{|c|c|c|c|c|c|c|}
\hline FORMIGA: S (30) & FAMÍLIA & Or. & Pr. & $\begin{array}{c}N \\
(43)\end{array}$ & $\begin{array}{l}\text { FR } \\
(\%)\end{array}$ & $\begin{array}{l}\text { FAc } \\
(\%)\end{array}$ \\
\hline Leucaena leucocephala (Lam.) R. de Wit - Leucena & Fabaceae & $\mathrm{E}$ & $\mathrm{M}$ & 5 & 11,6 & 11,6 \\
\hline Schinus molle L. - Chorão & Anarcadiaceae & $\mathrm{N}$ & $\mathrm{M}$ & 4 & 9,3 & 20,9 \\
\hline Terminalia argentea Mart. - Capitão-do-mato & Combretaceae & $\mathrm{N}$ & G & 2 & 4,7 & 25,6 \\
\hline Copaifera langsdorffii Desf. - Copaíba & Fabaceae & $\mathrm{N}$ & $\mathrm{G}$ & 2 & 4,7 & 30,2 \\
\hline $\begin{array}{l}\text { Handroanthus heptaphyllus (Vell.) Toledo - Ipê-roxo-de-sete- } \\
\text { folhas }\end{array}$ & Bignoniaceae & $\mathrm{N}$ & G & 2 & 4,7 & 34,9 \\
\hline Mangifera indica L. - Mangueira & Anacardiaceae & $\mathrm{E}$ & G & 2 & 4,7 & 39,5 \\
\hline Bauhinia forficata Link - Mororó-de-espinho & Fabaceae & $\mathrm{N}$ & G & 2 & 4,7 & 44,2 \\
\hline Licania tomentosa (Benth.) Fritsch - Oiti & Chrysobalanaceae & $\mathrm{N}$ & G & 2 & 4,7 & 48,8 \\
\hline Persea americana Mill. - Abacateiro & Lauraceae & $\mathrm{E}$ & G & 1 & 2,3 & 51,1 \\
\hline Eriobothrya japonica (Thunb.) Lindl. - Ameixa-nêspera & Rosaceae & $\mathrm{E}$ & M & 1 & 2,3 & 53,5 \\
\hline Annona crassiflora Mart. - Araticum-do-campo & Annonaceae & $\mathrm{N}$ & $\mathrm{G}$ & 1 & 2,3 & 55,8 \\
\hline Annona neosalicifolia $\mathrm{H}$. Rainer - Araticum-falso & Annonaceae & $\mathrm{N}$ & G & 1 & 2,3 & 58,1 \\
\hline Cassia fistula L. - Cássia-chuva-de-ouro & Fabaceae & $\mathrm{E}$ & G & 1 & 2,3 & 60,5 \\
\hline Ficus microcarpa L. f. - Ficus-microcarpa & Moraceae & $\mathrm{E}$ & G & 1 & 2,3 & 62,8 \\
\hline Delonix regia (Bojer ex Hook) Raf. - Flamboyant & Fabaceae & $\mathrm{E}$ & G & 1 & 2,3 & 65,1 \\
\hline Psidium guajava L. - Goiabeira & Myrtaceae & $\mathrm{N}$ & M & 1 & 2,3 & 67,4 \\
\hline Astronium fraxinifolium Schott ex Spreng. - Gonçalo-alves & Anarcadiaceae & $\mathrm{N}$ & $\mathrm{G}$ & 1 & 2,3 & 69,8 \\
\hline Handroanthus serratifolius (Vahl) S. O. Grose - Ipê-amarelo & Bignoniaceae & $\mathrm{N}$ & G & 1 & 2,3 & 72,1 \\
\hline Tecoma stans (L.) Juss ex Kunth - Ipê-mirim & Bignoniaceae & $\mathrm{E}$ & $\mathrm{M}$ & 1 & 2,3 & 74,4 \\
\hline $\begin{array}{l}\text { Handroanthus impetiginosus (Mart. ex Tul.) L.P.Queiroz - Ipê- } \\
\text { roxo-de-bola }\end{array}$ & Bignoniaceae & $\mathrm{N}$ & G & 1 & 2,3 & 76,7 \\
\hline Artocarpus heterophyllus Lam. - Jaqueira & Moraceae & $\mathrm{E}$ & G & 1 & 2,3 & 79,1 \\
\hline Hymenaea martiana Hayne - Jatobá-miúdo & Fabaceae & $\mathrm{N}$ & G & 1 & 2,3 & 81,4 \\
\hline Zanthoxylum riedelianum Engl. - Mamica-de-porca & Rutaceae & $\mathrm{N}$ & G & 1 & 2,3 & 83,7 \\
\hline Ceiba speciosa (A.St.-Hil.) Ravenna - Paineira & Malvaceae & $\mathrm{N}$ & G & 1 & 2,3 & 86,1 \\
\hline Tibouchina granulosa (Desr.) Cogn. - Quaresmeira & Melastomataceae & $\mathrm{N}$ & G & 1 & 2,3 & 88,4 \\
\hline Sapindus saponaria L. - Saponária & Sapindaceae & $\mathrm{N}$ & G & 1 & 2,3 & 90,7 \\
\hline Terminalia catappa L. - Sete-copas & Combretaceae & $\mathrm{E}$ & G & 1 & 2,3 & 93,0 \\
\hline Poincianella pluviosa (DC.) L. P. Queiroz - Sibipiruna & Fabaceae & $\mathrm{N}$ & G & 1 & 2,3 & 95,4 \\
\hline Tamarindus indica L. - Tamarindo & Fabaceae & $\mathrm{E}$ & G & 1 & 2,3 & 97,7 \\
\hline Triplaris sp. - Triplaris & Polygonaceae & $\mathrm{N}$ & G & 1 & 2,3 & 100,0 \\
\hline ITAÚNA: S (8) & FAMÍLIA & Or. & Pr. & $\begin{array}{c}\mathrm{N} \\
(\mathbf{2 8})\end{array}$ & $\begin{array}{l}\text { FR } \\
(\%)\end{array}$ & $\begin{array}{l}\text { FAc } \\
(\%)\end{array}$ \\
\hline Poincianella pluviosa (DC.) L. P. Queiroz - Sibipiruna & Fabaceae & $\mathrm{N}$ & G & 11 & 39,3 & 39,3 \\
\hline Schinus molle L. - Chorão & Anarcadiaceae & $\mathrm{N}$ & $\mathrm{M}$ & 5 & 17,9 & 57,1 \\
\hline Lafoensia glyptocarpa Koehne - Mirindiba & Lythraceae & $\mathrm{N}$ & G & 5 & 17,9 & 75,0 \\
\hline Murraya paniculata (L.) Jacq. - Murta & Rutaceae & $\mathrm{E}$ & $\mathrm{P}$ & 3 & 10,7 & 85,7 \\
\hline Ligustrum lucidum W. T. Aiton - Alfeneiro & Oleaceae & $\mathrm{E}$ & $\mathrm{M}$ & 1 & 3,6 & 89,3 \\
\hline Eriobothrya japonica (Thunb.) Lindl. - Ameixa-nêspera & Rosaceae & $\mathrm{E}$ & M & 1 & 3,6 & 92,9 \\
\hline Myroxylon peruiferum L. f. - Bálsamo & Fabaceae & $\mathrm{N}$ & G & 1 & 3,6 & 96,4 \\
\hline Colubrina glandulosa Perkins - Sobrasil & Rhamnaceae & $\mathrm{N}$ & $\mathrm{G}$ & 1 & 3,6 & 100,0 \\
\hline
\end{tabular}


Tabela 1. continuação.

\begin{tabular}{|c|c|c|c|c|c|c|}
\hline PARÁ DE MINAS: S (9) & FAMÍLIA & Or. & Pr. & $\begin{array}{c}\mathbf{N} \\
(\mathbf{3 1})\end{array}$ & $\begin{array}{c}\text { FR } \\
(\%)\end{array}$ & $\begin{array}{l}\text { FAc } \\
(\%)\end{array}$ \\
\hline Poincianella pluviosa (DC.) L. P. Queiroz - Sibipiruna & Fabaceae & $\mathrm{N}$ & $\mathrm{G}$ & 16 & 51,6 & 51,6 \\
\hline Sapindus saponaria L. - Saponária & Sapindaceae & $\mathrm{N}$ & $\mathrm{G}$ & 4 & 12,1 & 64,5 \\
\hline Spathodea campanulata P. Beauv. - Espatódea & Bignoniaceae & $\mathrm{E}$ & G & 3 & 9,7 & 74,2 \\
\hline Libidibia ferrea (Mart.) L. P. Queiroz - Pau-ferro & Fabaceae & $\mathrm{N}$ & $\mathrm{G}$ & 3 & 9,7 & 83,9 \\
\hline Acosmium subelegans (Mohlenbr.) Yakovlev - Amendoim-falso & Fabaceae & $\mathrm{N}$ & $\mathrm{P}$ & 1 & 3,2 & 87,1 \\
\hline Delonix regia (Bojer ex Hook) Raf. - Flamboyant & Fabaceae & $\mathrm{E}$ & $\mathrm{G}$ & 1 & 3,2 & 90,3 \\
\hline Platypodium elegans Vogel - Jacarandá-branco & Fabaceae & $\mathrm{N}$ & $\mathrm{G}$ & 1 & 3,2 & 93,6 \\
\hline Murraya paniculata (L.) Jacq. - Murta & Rutaceae & $\mathrm{E}$ & $\mathrm{P}$ & 1 & 3,2 & 96,8 \\
\hline Vitex polygama Cham. - Tarumã-do-Cerrado & Lamiaceae & $\mathrm{N}$ & $\mathrm{G}$ & 1 & 3,2 & 100,0 \\
\hline
\end{tabular}

Dados: $\mathrm{S}=$ quantidade de espécies; Or. = Origem; $\mathrm{E}=$ espécie exótica; $\mathrm{N}=$ espécie nativa; $\mathrm{Pr}$. = porte; $\mathrm{P}=$ pequeno porte $(\mathrm{P} \leq 6 \mathrm{~m}) ; \mathrm{M}$ $=$ médio porte $(6 \mathrm{~m}<\mathrm{M} \leq 10 \mathrm{~m}$ e grande porte); $\mathrm{G}=$ grande porte $(\mathrm{G}>10 \mathrm{~m}) ; \mathrm{N}=$ quantidade de indivíduos avaliados, $\mathrm{FR}=$ frequência relativa e FAc $=$ frequência acumulada.

A maior parte das árvores estava em ruas com largura acima de $7 \mathrm{~m}(84,8 \%)$, fator positivo relacionado à acessibilidade urbana de pessoas no conjunto de cidades amostrado. Em conformidade com Pivetta \& Silva Filho (2002), larguras de ruas maiores que $7 \mathrm{~m}$ são adequadas e proporcionam bom fluxo e acessibilidade. Por outro lado, aproximadamente um terço das árvores $(36,6 \%)$ estava localizado em calçadas com largura satisfatória (mínimo de $2 \mathrm{~m}$ ). O mesmo índice foi encontrado quando se avaliou a altura da primeira bifurcação, que deveria ser no mínimo $1,80 \mathrm{~m}$, altura que viabiliza o livre acesso a pedestres e cadeirantes.

Deste modo, os municípios devem priorizar intervenções, onde possíveis, como podas de levantamento de copa, buscando a adequação às normas regulamentadoras de acessibilidade urbana. Ainda, em novos plantios, é importante considerar a altura mínima da primeira bifurcação, para a aquisição de mudas de qualidade para projetos de arborização.

Quanto aos problemas fitossanitários relacionados ao tronco, percebeu-se que apenas quatro indivíduos de Schinus molle apresentaram estrangulamento (todos localizados na cidade de Itaúna), devido ao uso de grades de proteção de mudas no início do seu desenvolvimento.

Em relação à presença de rachaduras no tronco, 20 indivíduos foram afetados, sendo Ligustrum lucidum a espécie mais afetada, com registro em 14 indivíduos.
Quanto aos problemas relativos ao sistema radicular, atenção deve ser dada àqueles indivíduos que causaram levantamento de calçadas e exposição de raízes, sendo um indicador de que a espécie é inadequada àquele espaço, pois apresenta porte inadequado e sistema radicular agressivo. Foram registrados 87 indivíduos associados a esse problema, sendo três espécies mais representativas: Poincianella pluviosa (39 indivíduos), Ligustrum lucidum (17 indivíduos) e Michelia champaca (11 indivíduos). Além disso, Poincianella pluviosa apresentou oito, dos dez registrados com exposição de raízes.

Os indivíduos que sofreram alguma modalidade de poda perfizeram $71,2 \%$ da população amostral. As podas por causa de conflitos com a rede elétrica equivaleram a $40,2 \%$. As podas unilaterais e "em V" foram todas em decorrência da compatibilização da árvore com a rede elétrica (20,7\%), enquanto que as podas de condução ou formação e destopa foram responsáveis por $19,6 \%$ das podas relativas à compatibilização com a rede elétrica. As espécies mais podadas foram Michelia champaca e Poincianella pluviosa em Arcos, Itaúna e Pará de Minas, Ligustrum lucidum em Divinópolis e Leucaena leucocephala em Formiga.

As árvores com altura $\geq 6 \mathrm{~m}$ representaram $72,8 \%$ da população amostral (Figura 2). Esta característica é considerada um fator de risco, pois são árvores potenciais ao contato com a rede elétrica, podendo causar desligamentos, caso não sejam corretamente podadas ou conduzidas. 


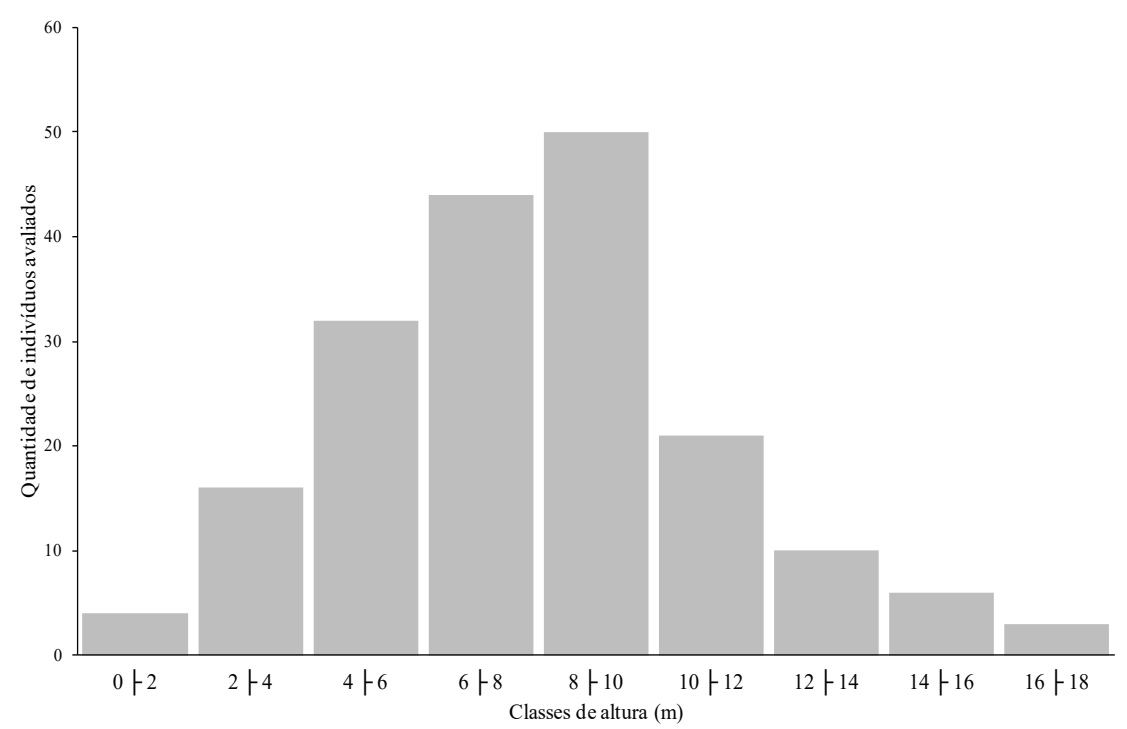

Figura 2. Classes de altura para os indivíduos sob rede elétrica avaliadas em cinco cidades da regional Oeste de Minas Gerais.

Das árvores com altura potencial para gerar conflitos com a rede elétrica, a maioria (56 indivíduos - 27,4\%) era de Poincianella pluviosa, de origem nativa, mas de grande porte e inadequada para o plantio sob redes elétricas.

Com a estratificação da localização das espécies, de acordo com o circuito elétrico amostrado (Tabela 2), foi possível perceber o grau de contribuição na riqueza e diversidade de espécies de cada circuito para o total de cada cidade.

$\mathrm{O}$ circuito $\mathrm{F} 3$ foi o de maior destaque, com 20 indivíduos classificados em 19 espécies e apresentou o maior valor no índice de riqueza de Odum $(6,34)$ e de diversidade de Shannon-Wiener $(2,93)$, porém no limiar inferior do valor três recomendado conforme Paiva ( 2009). Este fato decorre do local amostrado, que era um bairro externo à cidade, num condomínio fechado, em que os moradores fizeram um plantio com alternância de espécies. O interessante é que se obtenha o maior índice de equabilidade de Pielou $(0,99)$, circuito considerado com uma distribuição bastante heterogênea, ou seja, com apenas uma espécie que apresentou dois indivíduos e o restante com um indivíduo para cada espécie.
Tabela 2. Quantidade de indivíduos (N) e espécies (S), índices de riqueza de Odum (D), diversidade de espécies de ShannonWiener (H') e equabilidade de Pielou (J') para as cidades e circuitos amostrados da regional Oeste de Minas Gerais, em janeiro de 2013.

\begin{tabular}{lcccc}
\hline $\begin{array}{c}\text { Cidades e circuitos elétricos } \\
\text { amostrados }\end{array}$ & (N)-(S) & D & H' & J' \\
\hline Arcos & $48-7$ & 1,81 & 1,27 & 0,65 \\
A1) CF 36527 & $24-4$ & 1,26 & 0,78 & 0,56 \\
A2) CF 37441 & $24-5$ & 1,57 & 1,41 & 0,87 \\
\hline Divinópolis & $36-12$ & 3,35 & 1,78 & 0,72 \\
D1) T 28965-3-75 & $18-11$ & 3,81 & 2,18 & 0,91 \\
D2) T 5024-3-112,5 & $18-2$ & 0,69 & 0,21 & 0,31 \\
\hline Formiga & $43-30$ & 7,98 & 3,25 & 0,96 \\
F1) CF 118990 & $15-7$ & 2,58 & 1,77 & 0,91 \\
F2) CF 36693 & $8-6$ & 2,89 & 1,73 & 0,97 \\
F3) CF 36721 & $20-19$ & 6,34 & 2,93 & 0,99 \\
\hline Itaúna & $28-8$ & 2,40 & 1,70 & 0,82 \\
I1) T 1210-3-75 & $28-8$ & 2,40 & 1,70 & 0,82 \\
Pará de Minas & $31-9$ & 2,62 & 1,61 & 0,73 \\
PM1) CF 75234 & $18-3$ & 1,04 & 0,43 & 0,39 \\
PM2) T 10218-3-45 & $13-6$ & 2,34 & 1,63 & 0,91 \\
\hline Total Geral & $186-47$ & 8,99 & 2,95 & 0,77 \\
\hline
\end{tabular}

Nota: Dispositivos amostrados: CF (chave fusível) e T (transformador). Endereços dos circuitos amostrados: A1 (Rua Vereador João Veloso, 858), A2 (Rua São Geraldo, 548), D1 (Rua Mato Grosso, 1536), D2 (Rua Rubi, 283), F1 (Rua Hortência Rodrigues Oliveira, 115), F2 (Av. Geraldo Almeida, 1580), F3 (Rural Folha 41-16-03), I1 (Av. Magalhães Pinto, 608), PM1 (Rua Epaminondas Marinho, 150), PM2 (Av. Ovídio de Abreu, 277). 
A análise de todos os valores de diversidade de Shannon-Wiener encontrados para os circuitos (Tabela 2) mostrou que somente a cidade de Formiga apresentou um valor considerável, ou seja, recomendado por ser maior que três $(3,25)$. Quanto à adequação das espécies à rede elétrica, ou seja, espécies com porte até seis $m$ de altura, apenas quatro eram adequadas à compatibilização com a fiação, pois eram de pequeno porte (Nerium oleander, Lagerstroemia indica, Caesalpinia pulcherrima e Acosmium subelegans).

De acordo com Silva Filho \& Bortoleto (2005) para a arborização viária sob rede devem ser utilizadas espécies adequadas ao plantio sob fiação. De nada adiantaria ter uma diversidade máxima em um dado circuito (rua, bairro e cidade), se não considerar a adequação das espécies ao seu local de plantio, podendo localizar-se sob redes, fora da rede em ruas com largura inferior a $7 \mathrm{~m}$ (pequeno e médio porte), fora da rede com largura superior a $7 \mathrm{~m}$ (todos os portes), praças, parques, lotes vagos, barrancos, dentre outros.

$\mathrm{O}$ circuito D2 foi o que apresentou o menor índice de riqueza de Odum $(0,69)$, com baixa intensidade de mistura de espécies, o que não provoca grandes incertezas; isso porque foram encontradas somente duas espécies, Ligustrum lucidum (17 indivíduos) e Licania tomentosa (um indivíduo). Logo, o índice de diversidade de Shannon-Wiener $(0,21)$ foi baixo e, em relação ao índice de equabilidade de Pielou $(0,31)$, em razão das

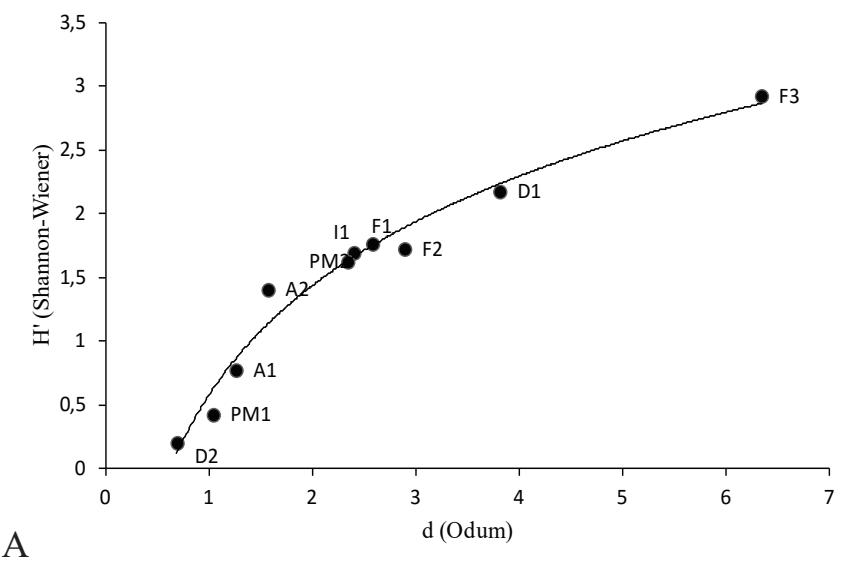

poucas espécies amostradas, houve baixa uniformidade, concentrando a maior parte dos indivíduos na espécie Ligustrum lucidum e apenas um em Licania tomentosa.

Verificou-se que houve correlação significativa entre os índices de riqueza de espécies de Odum e de diversidade de Shannon-Wiener para os dez circuitos amostrados (Figura 3), o que demonstrou que as espécies apresentaram distribuição aleatória no conjunto avaliado, segundo metodologia aplicada por Silva Filho \& Bortoleto (2005).

Em um plantio ordenado, incluído em um planejamento efetivo da arborização urbana, este não se mostraria correlacionado com os índices de riqueza de Odum e de diversidade de espécies de Shannon-Wiener (Figura 3b). O modelo gerado considerou a composição percentual de $10 \%$ para a distribuição de 15 diferentes espécies em um dado planejamento arbóreo urbano de 150 indivíduos no setor A, 300 indivíduos no setor B e 750 indivíduos no setor $C$. Não foi obtida correlação entre os índices de riqueza de Odum (d) e diversidade de espécies de Shannon-Wiener (H').

Um bom planejamento da arborização urbana leva em consideração, no mínimo, os valores percentuais das famílias, gêneros e espécies (Santamour Junior, 1990), podendo ser mais efetivo se levar em consideração o índice de Shannon-Wiener maior que três para a composição florística (Paiva, 2009) e espécies de pequeno porte sob redes elétricas, bem como espécies adequadas a outros locais da malha viária.

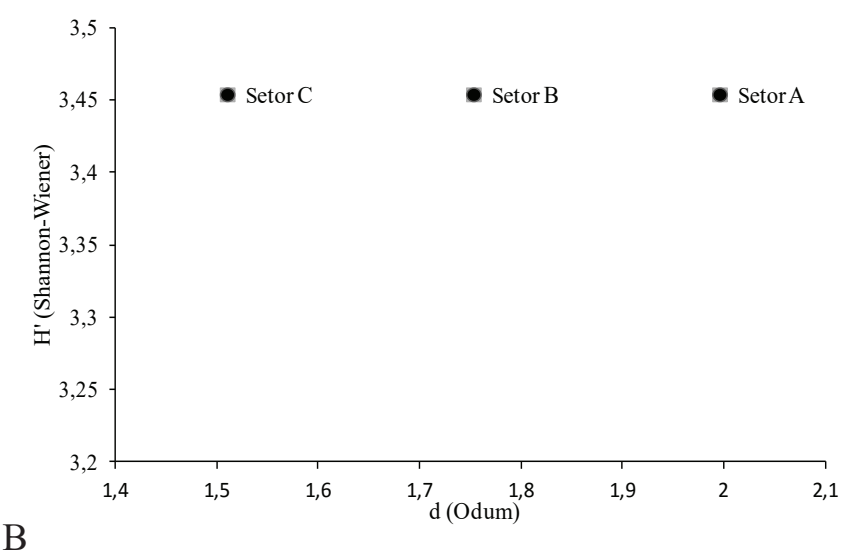

Figura 3. a) Correlação entre índice de riqueza de Odum (d) e diversidade de espécies de Shannon-Wiener (H') para os dez circuitos amostrados nas cinco cidades da regional Oeste de Minas Gerais. b) Arborização planejada para três setores ou bairros arborizados, hipoteticamente, segundo recomendação de Santamour Junior (1990). Nota: Valor r de Pearson =0,9331; $\mathrm{R}^{2}=$ 0,8707; valor de $\mathrm{p}=0,0003$, significativo em um intervalo de $95 \%$ de confiança, pelo teste $\mathrm{t}$. 


\section{Conclusão}

Nas cidades avaliadas não há riqueza e diversidade de espécies adequadas ao convívio com a rede elétrica devido ao porte. Além disso, a maioria das espécies era de médio e grande porte, sugerindo não haver um planejamento adequado na implantação da arborização viária sob a rede elétrica nestas cidades. Recomenda-se que sejam ampliados os estudos quanto aos benefícios proporcionados pelas árvores de diferentes portes e espécies, o que facilitará o trabalho dos gestores da arborização urbana.

\section{Referências}

AGUIRRE JUNIOR, J. H.; LIMA, A. M. L. P. Uso de árvores e arbustos em cidades brasileiras. Revista da Sociedade Brasileira de Arborização Urbana, Piracicaba, v. 2, n. 4, p. 50-66, 2007.

ARAUJO, A. C. B.; GRACIOLI, C. R.; GRIMM, E. L.; LONGHI, S. J. Avaliação da florística, do porte e da fitossanidade atual da arborização do Parque Internacional em Sant'ana do Livramento/ Rivera, Brasil/Uruguai. Revista da Sociedade Brasileira de Arborização Urbana, Piracicaba, v. 7, n. 1, p. 112-125, 2012.

BOBROWSKI, R. Estrutura e dinâmica da arborização de ruas de Curitiba-Paraná, no período 1984-2010. 2011. 144 f. Dissertação (Mestrado em Engenharia Florestal) - Universidade Federal do Paraná, Curitiba.

COMPANHIA ENERGÉTICA DE MINAS GERAIS. Manual de arborização. Belo Horizonte: CEMIG/Fundação Biodiversitas, 2011. $111 \mathrm{p}$

COMPANHIA ENERGÉTICA DE MINAS GERAIS. Manual de distribuição: norma de distribuição-ND-2.1: instalações básicas de redes de distribuição aéreas urbanas. Belo Horizonte: CEMIG, 2002. $186 \mathrm{p}$

COMPANHIA ENERGÉTICA DE MINAS GERAIS. Relatório do sistema Gemini Cemig. Belo Horizonte: CEMIG, 2013.

CUPERTINO, M. A.; EISENLOHR, P. V. Análise florística comparativa da arborização urbana nos campi universitários do Brasil. Bioscience Journal, Uberlândia, v. 29, n. 3, p. 739-750, 2013.

DRISCOLL, A. N.; RIES, P. D.; TILT, J. H.; GANIO, L. M. Needs and barriers to expanding urban forestry programs: an assessment of community officials and program managers in te Portland - Vancouver metropolitan region. Urban Forestry and Urban Greening, v. 14, n. 1, p. 48-55, 2015. DOI: 10.1016/j.ufug.2014.11.004

DZHAMBOV, A. M.; DIMITROVA, D. D. Green spaces and environmental noise perception. Urban Forestry and Urban Greening, v. 14, n. 4, p. 1000-1008, 2015. DOI: 10.1016/j. ufug.2015.09.006.

Pesq. flor. bras., Colombo, v. 36, n. 85, p. 31-40, jan./mar. 2016
GRAPHPAD PRISM Software for Windows. Version 4.0. San Diego, California, USA. <www.graphpad.com>. Acesso em: 30 dez. 2015

IBGE. Dados estatísticos das cidades mineiras. Rio de Janeiro, 2010. Disponível em: <http://www.ibge.gov.br/cidadesat/xtras/ uf.php? coduf $=31 \&$ search $=$ minas-gerais $>$. Acesso em: 11 ago. 2013 .

LISTA de espécies da flora do Brasil. 2012. Disponível em: <http:// floradobrasil.jbrj.gov.br/2012>. Acesso em: 9 set. 2013.

MAGALHÃES, L. M. S. Arborização e florestas urbanasterminologia adotada para a cobertura arbórea das cidades brasileiras. Série Técnica Floresta e Ambiente, Seropédica, v. 1, p. 23-26, 2006.

MELAZO, G. C.; NISHIYAMA, L. Mapeamento da cobertura arbóreo-arbustiva em quatro bairros da cidade de Uberlândia, MG. Revista da Sociedade Brasileira de Arborização Urbana, Piracicaba, v. 5, n. 2, p. 52-66, 2010.

MILANO, M. S. Aspectos quali-quantitativos da arborização de ruas de Curitiba. In: CONGRESSO BRASILEIRO SOBRE ARBORIZAÇÃO URBANA, 1., 1992, Vitória. Anais... Vitória: Sociedade Brasileira de Arborização Urbana, 1992. p. 199-210.

MILANO, M. S. Planejamento e replanejamento de arborização de ruas. In: ENCONTRO NACIONAL SOBRE ARBORIZAÇÃO URBANA, 2., 1987, Maringá. Anais... Maringá: Prefeitura Municipal de Maringá, 1987. p. 1-8.

MINAS GERAIS. Zoneamento ecológico e econômico de Minas Gerais. Disponível em: <http://www.zee.mg.gov.br>. Acesso em: 20 ago. 2013.

PAIVA, A. V. Aspectos da arborização urbana do centro de Cosmópolis, SP. Revista da Sociedade Brasileira de Arborização Urbana, Piracicaba, v. 4, n. 4, p. 17-31, 2009.

PAIVA, H. N.; GONÇALVES, W. Florestas urbanas: planejamento para melhoria da qualidade de vida. Viçosa, MG: Aprenda fácil, 2002. 180 p. (Coleção Jardinagem e Paisagismo, 2).

PINTO-COELHO, R. M. Fundamentos em ecologia. Porto Alegre: Artmed, 2000. 252 p.

PIVETTA, K. F. L.; SILVA FILHO, D. F. Arborização urbana. Jaboticabal: UNESP/FCAV/FUNEP, 2002. 69 p. (Série Arborização Urbana).

SANTAMOUR JUNIOR, F. S. Trees for urban planting: diversity, uniformity, and common sense. In: CONFERENCE OF THE METROPOLITAN TREE IMPROVEMENT ALLIANCE, 7., 1990, Lisle. Proceedings...Lisle: METRIA, 1990. p.57-65.

SANTOS, N. R. Z.; TEIXEIRA, I. F. Arborização de vias públicas: ambiente x vegetação. Santa Cruz do Sul: Instituto Souza Cruz, 2001. 135 p.

SILVA FILHO, D. F.; BORTOLETO, S. Uso de indicadores de diversidade na definição de plano de manejo da arborização viária de Águas de São Pedro, SP. Revista Árvore, Viçosa, MG, v. 29, n. 6, p. 973-982, 2005.

THE PLANT LIST: A WORKING LIST OF ALL PLANT SPECIES. Disponível em: <http://www.theplantlist.org/>. Acesso em: 9 set. 2013. 